\title{
Relationship between cardiac deformation parameters measured by cardiovascular magnetic resonance and aerobic fitness in endurance athletes
}

Peter P. Swoboda1*, Bara Erhayiem', Adam K. McDiarmid', Rosalind E. Lancaster², Gemma K. Lyall²,

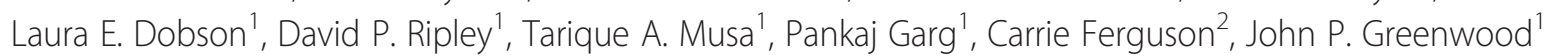
and Sven Plein ${ }^{1}$

\begin{abstract}
Background: Athletic training leads to remodelling of both left and right ventricles with increased myocardial mass and cavity dilatation. Whether changes in cardiac strain parameters occur in response to training is less well established. In this study we investigated the relationship in trained athletes between cardiovascular magnetic resonance (CMR) derived strain parameters of cardiac function and fitness.

Methods: Thirty five endurance athletes and 35 age and sex matched controls underwent CMR at 3.0 T including cine imaging in multiple planes and tissue tagging by spatial modulation of magnetization (SPAMM). CMR data were analysed quantitatively reporting circumferential strain and torsion from tagged images and left and right ventricular longitudinal strain from feature tracking of cine images. Athletes performed a maximal ramp-incremental exercise test to determine the lactate threshold (LT) and maximal oxygen uptake $\left(\mathrm{V}^{2} \mathrm{Imax}_{\text {max }}\right)$.

Results: LV circumferential strain at all levels, LV twist and torsion, LV late diastolic longitudinal strain rate, RV peak longitudinal strain and RV early and late diastolic longitudinal strain rate were all lower in athletes than controls. On multivariable linear regression only LV torsion (beta $=-0.37, P=0.03$ ) had a significant association with LT. Only RV longitudinal late diastolic strain rate (beta $=-0.35, P=0.03$ ) had a significant association with $\dot{\mathrm{V}} \mathrm{O}_{2 \text { max }}$.

Conclusions: This cohort of endurance athletes had lower LV circumferential strain, LV torsion and biventricular diastolic strain rates than controls. Increased LT, which is a major determinant of performance in endurance athletes, was associated with decreased LV torsion. Further work is needed to understand the mechanisms by which this occurs.
\end{abstract}

Keywords: Cardiovascular magnetic resonance, Tissue tagging, Feature tracking, Athlete, Aerobic capacity, Lactate threshold

\footnotetext{
* Correspondence: p.swoboda@leeds.ac.uk

${ }^{1}$ Multidisciplinary Cardiovascular Research Centre (MCRC) and Leeds Institute

of Cardiovascular and Metabolic Medicine, University of Leeds, Clarendon

Way, Leeds LS2 9JT, UK

Full list of author information is available at the end of the article
} 


\section{Background}

It is well recognised that athletic training leads to ventricular remodelling, specifically increases in left and right ventricular end diastolic volume (LVEDV \& RVEDV) and left ventricular mass (LVM) [1, 2]. These structural changes are most frequently seen in athletes who undergo prolonged periods of endurance training [3].

Although less well established, there is also evidence for changes in functional parameters in the hearts of trained athletes. Endurance athletes have reduced ejection fraction, circumferential and longitudinal strain of both the left and right ventricles compared to healthy controls [4-6]. The heart has a complex twisting motion where the base rotates clockwise in early systole and the apex rotates anticlockwise in later systole. These opposing directions of rotation at the apex and base generate maximal torsional force at end systole [7]. It has been reported that athletes have decreased LV twist and torsion when compared to controls [5].

Both strain and torsion parameters can be measured using cardiovascular magnetic resonance (CMR) tissue tagging techniques [8]. CMR tagging is considered to be the gold standard for measurement of myocardial strain and torsion $[9,10]$. More recently, post-processing feature tracking of cine images has been proposed for quantification of strain without the need for acquisition of tagged CMR data [11]. Previous studies have shown good agreement between strain parameters derived from feature tracking and tissue tagging [11].

Cardiopulmonary exercise testing (CPX) with breathby-breath measurement of gas exchange responses is an established method for assessment of whole-body exercise tolerance, and key parameters of aerobic function: the lactate threshold (LT) and the maximal pulmonary oxygen uptake $\left(\dot{\mathrm{VO}}_{2 \max }\right)$ [12]. This information gives an accurate and reproducible measure of the integrated capacity of the respiratory, cardiovascular, and neuromuscular systems, and is frequently used to quantitatively assess aerobic capacity and training status [13]. Previous studies have demonstrated a clear correlation between LV remodelling and $\dot{\mathrm{VO}}_{2 \max }$ in endurance athletes [14-16].

In this study we investigated the relationship between strain-derived parameters of cardiac function and CPXderived performance parameters that has not previously been investigated. We hypothesised that strain parameters measured at rest would be lower in athletes than in controls and lowest in athletes with the highest $\dot{\mathrm{V}} \mathrm{O}_{2 \max }$. We also planned to specifically investigate if any strain parameters were associated with LT.

\section{Methods}

\section{Enrolment recruitment}

Thirty five endurance athletes were recruited from local sporting clubs. They all trained more than $6 \mathrm{~h}$ a week and competed regularly at local, national or international level. Exclusion criteria were any medical illness or contraindication to CMR. Thirty five controls who exercised less than $3 \mathrm{~h}$ a week were also recruited and prospectively matched to the athletes for age and gender. No athletes or controls had any medical condition or took any regular medication.

\section{CMR protocol}

CMR was performed on a dedicated cardiovascular 3 Tesla Philips Achieva system equipped with a 32 channel coil and MultiTransmit ${ }^{\circledR}$ technology. Data was acquired during breath-holding at end expiration. From scout CMR images, the left ventricular long and short axes were determined.

Cine images covering the entire heart in the LV short axis plane were acquired (balanced steady state free precession (SSFP), spatial resolution $1.2 \times 1.2 \times 10 \mathrm{~mm}^{3}, 30$ cardiac phases TR/TE $2.6 / 1.3 \mathrm{~ms}$, flip angle $40^{\circ}$, field of view 300-420 mm, typical temporal resolution $39 \mathrm{~ms}$ ) and in orthogonal long-axis planes. Then axial cine images planned to cover the right ventricle were acquired (balanced SSFP, spatial resolution $1.2 \times 1.2 \times 6 \mathrm{~mm}^{3}, 30$ cardiac phases TR/TE $2.6 / 1.3 \mathrm{~ms}$, flip angle $40^{\circ}$, field of view 300-420 mm).

Tissue tagging by spatial modulation of magnetization (SPAMM) (spatial resolution $1.51 \times 1.57 \times 10 \mathrm{~mm}^{3}$, tag separation $7 \mathrm{~mm}, \geq 18$ phases, typical TR/TE $5.8 / 3.5 \mathrm{~ms}$, flip angle $10^{\circ}$, typical temporal resolution $55 \mathrm{~ms}$ ) was acquired in the three short axis slices acquired at the apex, mid-ventricle, and base. Slices were positioned using the highly reproducible "3 of 5 technique" [17].

\section{Image analysis}

CMR data were analysed quantitatively using commercially available software (CVI42, Circle Cardiovascular Imaging Inc. Calgary, Canada and inTag v1.0, CREATIS lab, Lyon, France). Epicardial and endocardial borders were traced on the LV and RV cine stack at end-diastole and end-systole to calculate end diastolic volume (LVEDV), end systolic volume (ESV), stroke volume (SV), ejection fraction (EF) and LV mass. Volumes were indexed to body surface area (BSA) calculated using the Mosteller equation.

For tagging analysis endocardial and epicardial contours were drawn on the short axis SPAMM sequences using a semi-automated process. Peak circumferential LV strain was measured for the three slices at apex, mid-ventricle, and base. Peak systolic and both early and late diastolic LV strain rates were measured from the mid-ventricular slice. Strain was measured in the mid-myocardial layer which has previously been reported to be the most reproducible [18]. LV twist was calculated by subtracting the basal from apical rotation. Basal and apical radius was 
calculated from cine images in diastole at the same slice location as the tagged images. The equation used to determine torsion was [10]:

$$
\text { Torsion }=\frac{\text { Peak Twist } \times(\text { Apical Radius }+ \text { Basal Radius })}{2 \times \text { Apex to Base length }}
$$

For feature tracking analysis endocardial and epicardial contours were drawn on a long axis 4 chamber cine using a semi-automated process. Peak longitudinal strain, systolic strain rate (SSR), early and late diastolic strain rates (EDSR and LDSR) were measured for both the LV \& RV. LDSR was defined as peak rate during atrial contraction. We have used feature tracking rather than SPAMM for the analysis of longitudinal strain. However, tissue tagging is hampered by a lower temporal resolution than cine imaging and tag fading during diastole. As we specifically wanted to examine longitudinal strain rates in diastole we therefore chose to use feature tracking for this while using SPAMM tagging for assessment of circumferential strain parameters.

\section{Exercise protocol}

A ramp-incremental test $(20-30 \mathrm{~W} / \mathrm{min})$ to the limit of tolerance was performed on a cycle ergometer (Excalibur Sport, Lode BV, Groningen, the Netherlands), with breathby-breath pulmonary gas exchange measured throughout (Cardio2, Medgraphics, Medical Graphics Corporation, St Paul, MN, USA). A 12-lead ECG was also monitored throughout this test, with heart rate determined from the $\mathrm{R}-\mathrm{R}$ interval. LT was estimated using standard ventilatory and pulmonary gas-exchange criteria (REF), and $\dot{\mathrm{VO}}_{2 \max }$ determined as the highest 12-breath mean. An additional step-exercise test performed to the limit of tolerance confirmed that $\dot{\mathrm{V}}_{2 \max }$ was attained in all participants [19].

\section{Statistical analysis}

Statistical analysis was performed using IBM SPSS $^{\circ}$ Statistics 20.0 (IBM Corp., Armonk, NY). Continuous variables were expressed as means $\pm \mathrm{SD}$. Categorical variables were expressed as $N(\%)$. Shapiro-Wilk test was used to test normality and unpaired t-tests and Mann Whitney $U$ test used to compare athletes and controls. Pearson's coefficient was used to measure correlation between exercise and CMR parameters. Univariable analyses were performed to identify predictors of $\mathrm{LT}$ and $\dot{\mathrm{VO}}_{2 \max }$. Variables with a probability value $<0.1$ in the univariable analysis were included in a multivariable linear regression analysis. The standardised coefficient (beta) is reported. $P<0.05$ was considered statistically significant.

\section{Results}

Study participant demographics and characteristics Of 35 athletes 7 were runners, 15 cyclists and 13 triathletes. The athletes trained $11.5 \pm 3.7 \mathrm{~h}$ per week and all had trained $>6 \mathrm{~h}$ per week for $8.4 \pm 6.0$ years. Mean ramp duration was $772 \pm 93 \mathrm{~s}$ reaching a peak work rate of $370 \pm 64 \mathrm{~W}$. HR rose from $55 \pm 7$ beats/min at rest to $182 \pm 10$ beats/min at peak exercise. Mean LT was 2.60 $\pm 0.57 \mathrm{l} / \mathrm{min}, 36.5 \pm 6.7 \mathrm{ml} / \mathrm{min} / \mathrm{kg}$ (normalised to body weight) or $62.1 \pm 8.0 \% \dot{\mathrm{VO}}_{2 \max }$. Mean $\dot{\mathrm{VO}}_{2 \max }$ was $4.2 \pm$ $0.80 \mathrm{l} / \mathrm{min}, 58.9 \pm 8.2 \mathrm{ml} / \mathrm{min} / \mathrm{kg}$ or $160.0 \pm 18.8 \%$ of predicted $\dot{\mathrm{V}}_{2 \max }[20]$.

Athletes and controls were prospectively matched for age and gender (Table 1). BMI and resting heart rate were lower in athletes than controls $(P=0.001$ and $P<0.001$ respectively).

\section{CMR findings}

LV volumes for athletes and controls are shown in Table 2. LVEDV, LVM and RVEDV indexed to BSA were greater in athletes than controls. LVEF was lower in athletes than controls $(P=0.04)$ but there was no difference in $\operatorname{RVEF}(P=0.27)$. Strain parameters are shown in Table 3. LV circumferential strain at all levels, LV twist and torsion (Fig. 1), LV longitudinal LDSR (Fig. 2), RV peak longitudinal strain and RV EDSR and LDSR were all lower in athletes than controls.

In athletes, there were no significant correlations between left ventricle mass indexed to BSA (LVMI), left ventricle end diastolic volume indexed to BSA (LVEDVI) and LVM/EDV and LV twist $(P=0.20,0.85$ and 0.21 respectively). LV torsion had a trend to correlation with LVMI $(R=-0.34, P=0.05)$ but there were no significant associations with LVEDVI or LVM/EDV $(P=0.61$ and 0.08 respectively).

\section{Relationship between CPX and functional CMR parameters in athletes}

The only significant correlations with LT $\left(\% \dot{\mathrm{V}}{ }_{2 \max }\right)$ were with torsion parameters. Peak twist $(r=-0.45, P=0.01)$, peak torsion $(r=-0.36, P=0.04)$ and twist rate $(r=-0.38$,

Table 1 Subject characteristics

\begin{tabular}{lccc}
\hline & Athlete & Control & $P$ value \\
\hline Age & $31.3 \pm 7.6$ & $30.6 \pm 8.5$ & 0.72 \\
Male, \% & $27(77)$ & $27(77)$ & 1.0 \\
Height, cm & $178.7 \pm 8.7$ & $176.5 \pm 8.2$ & 0.29 \\
Weight, kg & $71.4 \pm 9.9$ & $77.0 \pm 14.8$ & 0.07 \\
BMl, $\mathrm{kg} / \mathrm{m}^{2}$ & $22.3 \pm 1.9$ & $24.5 \pm 3.3$ & 0.001 \\
HR & $55.0 \pm 6.5$ & $65.1 \pm 8.7$ & $<0.001$ \\
SBP, $\mathrm{mmHg}$ & $118.8 \pm 8.7$ & $114.7 \pm 10.6$ & 0.16 \\
DBP, $\mathrm{mmHg}$ & $71.0 \pm 9.2$ & $59.2 \pm 10.6$ & $<0.001$ \\
\hline
\end{tabular}


Table 2 CMR measured volumetric parameters

\begin{tabular}{lccc}
\hline & Athlete & Control & $P$ value \\
\hline Left ventricle & & & \\
EDV, $\mathrm{ml}$ & $217.1 \pm 34.8$ & $176.5 \pm 34.8$ & $<0.001$ \\
EDVI, $\mathrm{ml} / \mathrm{m}^{2}$ & $115.4 \pm 14.2$ & $90.8 \pm 12.9$ & $<0.001$ \\
ESV, ml & $96.1 \pm 18.7$ & $74.7 \pm 18.7$ & $<0.001$ \\
Ejection Fraction, \% & $55.7 \pm 4.5$ & $57.9 \pm 4.1$ & 0.04 \\
LVM, g & $127.9 \pm 24.6$ & $100.5 \pm 23.4$ & $<0.001$ \\
LVMI, g/m ${ }^{2}$ & $67.8 \pm 9.9$ & $51.5 \pm 9.1$ & $<0.001$ \\
LVM/EDV, g/ml & $0.59 \pm 0.07$ & $0.57 \pm 0.08$ & 0.25 \\
Right ventricle & & & \\
EDV, ml & $219.7 \pm 37.2$ & $204.8 \pm 50.1$ & 0.16 \\
EDVI, ml/m ${ }^{2}$ & $116.8 \pm 15.8$ & $105.1 \pm 19.7$ & 0.01 \\
ESV, ml & $104.2 \pm 22.7$ & $99.5 \pm 27.5$ & 0.44 \\
Ejection Fraction, \% & $52.8 \pm 4.7$ & $51.6 \pm 3.7$ & 0.27 \\
\hline
\end{tabular}

$P=0.03)$ all had a significant correlation with LT. There was no significant correlation between LT and any demographic, volumetric measurement (of those listed in Table 2) or other strain parameter. The decrease in LV twist and torsion was secondary to decreased apical rotation in the athletes with the highest LT (Fig. 3).

Table 3 CMR measured strain parameters

\begin{tabular}{|c|c|c|c|}
\hline & Athlete & Control & $P$ value \\
\hline \multicolumn{4}{|l|}{ LV Circumferential Strain } \\
\hline Apex, \% & $18.4 \pm 5.2$ & $23.4 \pm 4.9$ & $<0.001$ \\
\hline Mid LV, \% & $19.6 \pm 3.9$ & $21.5 \pm 2.5$ & 0.02 \\
\hline Base, $\%$ & $17.0 \pm 4.0$ & $20.5 \pm 2.5$ & $<0.001$ \\
\hline Systolic SR, \%/s & $115.3 \pm 12.8$ & $116.6 \pm 10.0$ & 0.66 \\
\hline Early diastolic SR,\%/s & $50.8 \pm 16.4$ & $51.0 \pm 16.0$ & 0.95 \\
\hline Late diastolic SR, \%/s & $140.1 \pm 40.7$ & $151.4 \pm 40.3$ & 0.27 \\
\hline \multicolumn{4}{|l|}{ Torsion } \\
\hline LV twist, $^{\circ}$ & $9.7 \pm 3.6$ & $13.3 \pm 3.8$ & $<0.001$ \\
\hline LV torsion, ${ }^{\circ}$ & $8.8 \pm 3.0$ & $11.9 \pm 3.1$ & $<0.001$ \\
\hline LV twist rate, $\% / s$ & $63.2 \pm 18.9$ & $72.4 \pm 27.8$ & 0.048 \\
\hline LV untwist rate, $\% / s$ & $88.1 \pm 25.5$ & $101.8 \pm 34.5$ & 0.07 \\
\hline \multicolumn{4}{|l|}{ LV Longitudinal Strain } \\
\hline Peak, \% & $17.1 \pm 2.8$ & $17.7 \pm 2.3$ & 0.30 \\
\hline $\mathrm{SSR}, \% / \mathrm{S}$ & $101.6 \pm 29.6$ & $103.2 \pm 19.8$ & 0.29 \\
\hline EDSR, \%/s & $90.6 \pm 32.4$ & $102.4 \pm 31.7$ & 0.13 \\
\hline LDSR, \%/s & $41.7 \pm 15.6$ & $57.3 \pm 19.6$ & $<0.001$ \\
\hline \multicolumn{4}{|l|}{ RV Longitudinal Strain } \\
\hline Peak, \% & $19.8 \pm 3.7$ & $22.6 \pm 3.4$ & 0.002 \\
\hline $\mathrm{SSR}, \% / \mathrm{S}$ & $137.7 \pm 49.9$ & $138.4 \pm 37.0$ & 0.50 \\
\hline EDSR, \%/s & $108.6 \pm 32.1$ & $124.6 \pm 32.9$ & 0.03 \\
\hline LDSR, \%/s & $69.2 \pm 40.2$ & $89.5 \pm 42.4$ & 0.02 \\
\hline
\end{tabular}

There was a correlation between $\dot{\mathrm{VO}}_{2 \max }$ (normalised to weight) and both LVMI $(r=0.59, P<0.001)$ and LVEDVI $(r=0.47, P=0.01)[14,21]$. There was also a trend to correlation between RVEDVI and $\dot{\mathrm{VO}}_{2 \max }(r=0.33, P=0.05)$. No LV strain parameters had a significant correlation with $\dot{\mathrm{V}}{ }_{2 \text { max }}$. RV longitudinal SSR $(r=-0.33, P=0.05)$ and RV LDSR $(r=-0.38, P=0.02)$ both correlated with $\dot{\mathrm{VO}}_{2 \max }$.

\section{Regression analysis}

On univariable linear regression of the parameters shown in Table 4 only LV torsion and sex were associated with LT. On multivariable linear regression only LV torsion (beta $=-0.37, P=0.03$ ) had a significant association with LT.

On univariable linear regression of the parameters shown in Table 5 age, sex, LVEDVI, LVMI, RVEDVI, RV longitudinal strain, RV longitudinal SSR and LDSR were associated with $\dot{\mathrm{VO}}_{2 \max }$. On multivariable linear regression only RV longitudinal LDSR (beta $=-0.39, P=0.03$ ) had a significant association with $\dot{\mathrm{VO}}_{2 \max }$.

\section{Discussion}

We have carried out comprehensive cardiac functional assessment of 35 endurance athletes from a broad spectrum of event type, age and athletic ability (range 123-206 \% predicted $\dot{\mathrm{VO}}_{2 \max }$ ). This diverse, but well characterised group has allowed us to investigate specifically the ventricular strain parameters that have a relationship with key parameters of aerobic function and exercise capacity, namely LT and $\dot{\mathrm{VO}}_{2 \max }[22]$.

\section{The relationship between LV torsion and lactate threshold}

The most striking finding was the inverse linear correlation between both LV twist and torsion, and LT. On multivariable linear regression no other factors significantly influenced LT. To our knowledge this is the first time a significant association between a cardiac structural or functional parameter and LT (key parameter of aerobic function) has been reported. Furthermore we have found that in athletes with the highest LT the decrease in torsion is secondary to decreased apical rotation.

We have reported with high statistical significance that LV torsion was lower in endurance athletes than controls. Previous CMR tagging studies have been small and insufficiently powered and therefore unable to report a difference in baseline torsion parameters between endurance athletes and controls [23, 24].

Several echocardiography studies using techniques including tissue Doppler imaging and speckle tracking have been used to investigate left ventricular torsion in athletes. Some have reported similar findings to ours of decreased apical rotation and LV torsion in athletes with high levels of aerobic fitness [25] whereas others have 

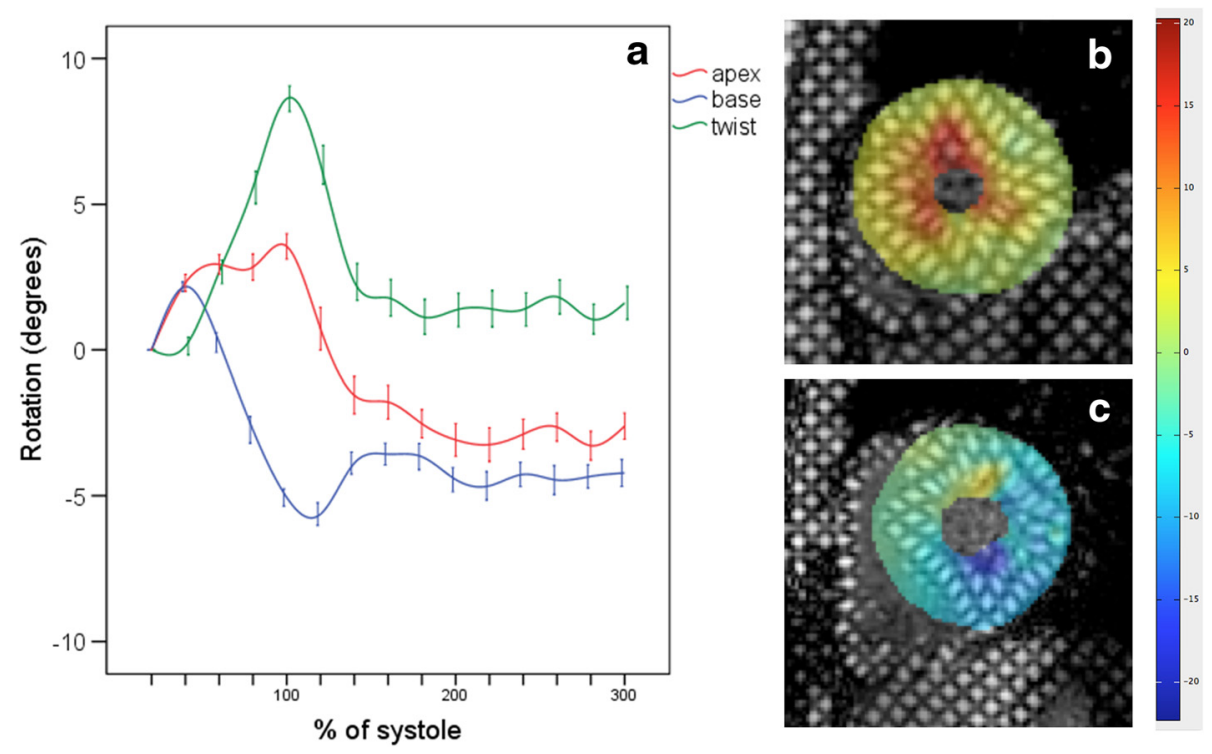

Fig. 1 Average apical rotation (red), basal (blue) rotation and twist (green) of the left ventricle of 35 endurance athletes (a). Each point represents mean rotation/twist and time in the cardiac cycle corrected to end-systole, error bars represent standard error of mean rotation/twist. Tagged images of anticlockwise apical (a) systolic rotation (yellow and red) and clockwise basal (c) rotation (green and blue)

reported that high intensity exercise either had no effect [26], or even lead to an increase [27] in LV torsion. The inconsistent results that have been reported may in part reflect different sport and training techniques, research methodology used and also the difficulty in positioning the apical and basal slices in echocardiography studies, which is based upon anatomical landmarks with a degree of subjectivity. In CMR on the other hand, positioning of the slices is carried out objectively based upon the length of the ventricle [17].

It has previously been suggested that decreased torsion in athletes is mediated by eccentric hypertrophy of the LV with decreased lever arm forces from epicardial fibres [28]. Athletes in the present study displayed eccentric hypertrophy (higher LVMI but LVM/EDV not significantly different than controls) despite this there
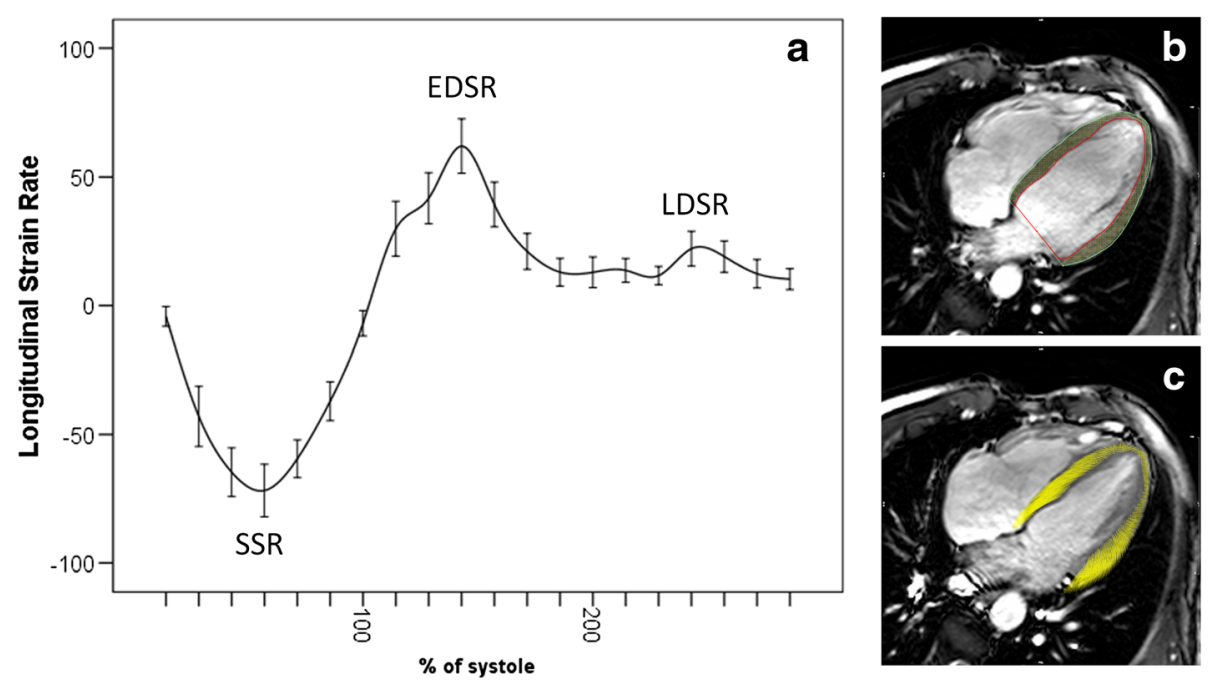

Fig. 2 Average LV longitudinal strain rate from 35 endurance athletes (a). Each point represents mean longitudinal strain rate at each point in the cardiac cycle corrected to end-systole, error bars represent $95 \%$ confidence interval of mean strain. Peak systolic strain rate (SSR), early diastolic strain rate (EDSR) and late diastolic strain rate (LDSR). SSFP cine image at end diastole showing manually drawn endocardial and epicardial contours (b). Feature tracked end systolic image (c) 

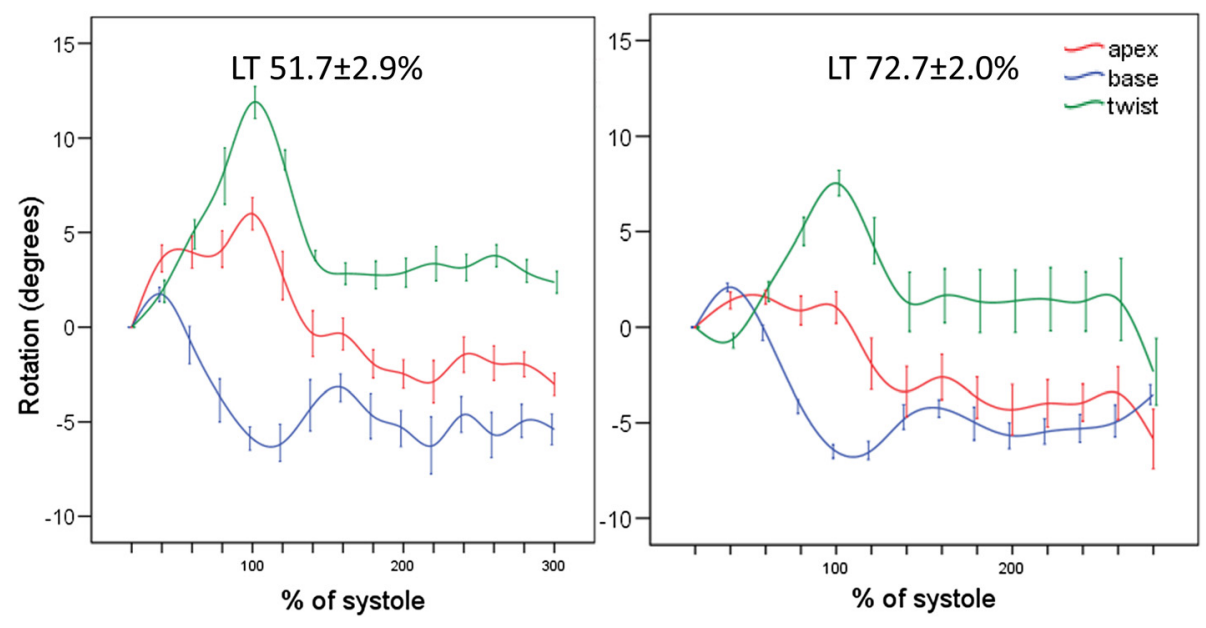

Fig. 3 Plots showing the mean \pm standard error of rotation/twist of the 8 athletes in the lowest quartile of LT (left) and 8 in the highest quartile of $L T$ (right). Peak twist was lower in athletes in the highest quartile of $L T$ ( $8.5 \pm 2.9 \mathrm{vs} .12 .9 \pm 2.6^{\circ} P=0.008$ ) which was secondary to a loss of apical rotation $\left(1.0 \pm 3.3\right.$ vs. $\left.6.0 \pm 3.1^{\circ} P<0.001\right)$. Basal rotation was not different between the groups $6.5 \pm 1.4$ vs. $6.1 \pm 2.6^{\circ} P=0.75$

was no significant association between twist or torsion and markers of remodelling such as LVM/EDV. Furthermore on linear regression analysis there was no association between LT and LVEDVI or LVMI. This suggests it is likely that the association between LT and decreased LV torsion is more complex than decreased lever arm forces.

Table 4 Univariable and multivariable linear regression analysis of factors with a significant association with lactate threshold

\begin{tabular}{|c|c|c|c|c|}
\hline & \multicolumn{2}{|c|}{ Univariable } & \multicolumn{2}{|c|}{ Multivariable } \\
\hline & Beta & $P$ value & Beta & $P$ value \\
\hline Age & -0.07 & 0.70 & & \\
\hline Sex & 0.29 & 0.09 & 0.29 & 0.08 \\
\hline LVMI, $g / \mathrm{m}^{2}$ & -0.01 & 0.98 & & \\
\hline LVEDVI, $\mathrm{ml} / \mathrm{m}^{2}$ & 0.15 & 0.39 & & \\
\hline LVEF, \% & -0.43 & 0.81 & & \\
\hline RVEF, \% & 0.10 & 0.56 & & \\
\hline RVEDI, $\mathrm{ml} / \mathrm{m}^{2}$ & 0.26 & 0.14 & & \\
\hline Apex circumferential strain, \% & 0.15 & 0.38 & & \\
\hline Mid LV circumferential strain, \% & -0.11 & 0.55 & & \\
\hline Base circumferential strain, \% & -0.05 & 0.77 & & \\
\hline LV torsion, ${ }^{\circ}$ & -0.36 & 0.04 & -0.37 & 0.03 \\
\hline LV longitudinal strain, \% & 0.09 & 0.61 & & \\
\hline LV longitudinal SSR, \%/s & 0.15 & 0.40 & & \\
\hline LV longitudinal EDSR, \%/s & -0.23 & 0.19 & & \\
\hline LV longitudinal LDSR, \%/s & -0.01 & 0.95 & & \\
\hline RV longitudinal strain, \% & -0.01 & 0.97 & & \\
\hline RV longitudinal SSR, \%/s & 0.04 & 0.84 & & \\
\hline RV longitudinal EDSR, \%/s & -0.01 & 0.97 & & \\
\hline RV longitudinal LDSR, \%/s & -0.22 & 0.20 & & \\
\hline
\end{tabular}

The trend to correlation between LVMI and LV torsion may reflect that as athletes train they increase both VO2max and LT (although different athletes and training programmes increase these two parameters at different rates). The training done to increase $\dot{\mathrm{V} O} 2 \mathrm{max}$ tends to be associated with an increase in LV mass [14-16] and we

Table 5 Univariable and multivariable linear regression analysis of factors with a significant association with $\dot{\mathrm{V}} \mathrm{O}_{2 \max }$

\begin{tabular}{|c|c|c|c|c|}
\hline & \multicolumn{2}{|c|}{ Univariable } & \multicolumn{2}{|c|}{ Multivariable } \\
\hline & Beta & $P$ value & Beta & $P$ value \\
\hline$\overline{\text { Age }}$ & -0.37 & 0.04 & -0.26 & 0.10 \\
\hline Sex & 0.40 & 0.02 & -0.30 & 0.14 \\
\hline LVMI, $g / \mathrm{m}^{2}$ & 0.59 & $<0.001$ & 0.19 & 0.37 \\
\hline LVEDVI, $\mathrm{ml} / \mathrm{m}^{2}$ & 0.47 & 0.01 & 0.16 & 0.48 \\
\hline LVEF, \% & -0.12 & 0.50 & & \\
\hline RVEF, \% & -0.52 & 0.77 & & \\
\hline RVEDI, $\mathrm{ml} / \mathrm{m}^{2}$ & 0.33 & 0.05 & 0.06 & 0.78 \\
\hline Apex circumferential strain, \% & 0.27 & 0.11 & & \\
\hline Mid LV circumferential strain, $\%$ & 0.18 & 0.92 & & \\
\hline Base circumferential strain, $\%$ & -0.17 & 0.33 & & \\
\hline LV torsion, ${ }^{\circ}$ & -0.21 & 0.24 & & \\
\hline LV longitudinal strain, \% & 0.17 & 0.32 & & \\
\hline LV longitudinal SSR, \%/s & 0.04 & 0.80 & & \\
\hline LV longitudinal EDSR, \%/s & -0.04 & 0.81 & & \\
\hline LV longitudinal LDSR, \%/s & -0.14 & 0.41 & & \\
\hline RV longitudinal strain, \% & 0.30 & 0.08 & -0.15 & 0.45 \\
\hline RV longitudinal SSR, \%/s & -0.33 & 0.05 & -0.24 & 0.14 \\
\hline RV longitudinal EDSR, \%/s & -0.01 & 0.96 & & \\
\hline RV longitudinal LDSR, \%/s & -0.38 & 0.02 & -0.35 & 0.03 \\
\hline
\end{tabular}


have reported that a higher LT is associated with decreased torsion.

An alternative mechanism for decreased torsion in athletes with high LT is cellular changes within the heart as a consequence of high-intensity training. It is well recognised that LT particularly as a proportion of $\dot{\mathrm{VO}}_{2 \max }$ is a key determinant of performance in endurance sport [29] and endurance athletes can increase their LT by regular high intensity training (such as interval training). Interval training has been associated with increased vascularity [30] and improved substrate utilisation [31] within the myocardium. It has also been reported that interval training leads to depressed LV ejection fraction, untwist rate, apical rotation rate and circumferential strain $30 \mathrm{~min}$ after a training session [23].

In diffuse myocardial diseases [32, 33] increased LV torsion is attributed to subendocardial microvascular hypoperfusion and contractile dysfunction. It is hypothesized that there is a compensatory increase in subepicardial fibre contraction giving rise to increased LV torsion yet unchanged overall circumferential strain. It is possible that the converse could be true in athletes with high intensity training leading to increased vascularity of the subendocardium. This would explain the correlation between LT and LV torsion but not measures of LV strain.

\section{The relationship between strain parameters and $\dot{\mathrm{VO}}_{2 \max }$}

We have also reported lower RV peak longitudinal strain, LV LDSR, RV EDSR and LDSR in athletes versus controls. On multivariable linear regression only increasing RV LDSR was associated with lower $\dot{\mathrm{VO}}_{2 \max }$. Although this result is less striking, to our knowledge it is the first time that CMR strain techniques have been used to compare longitudinal functional changes in athletes and controls. Conflicting findings have been reported when echocardiographic assessment of diastolic function has been used with some studies reporting augmented relaxation of both ventricles in endurance athletes $[4,34]$ and others reporting no difference from controls [35]. Our finding of reduced longitudinal LDSR of both ventricles of athletes and a negative association between longitudinal RV LDSR and $\dot{\mathrm{VO}}_{2 \max }$ can be attributed to reduced active atrial contraction late in diastole. In athletes the atrial contribution to ventricular filling is not required at rest but of course can then be utilised to maximise cardiac output during exercise.

\section{Limitations}

Our study was carried out on a cross sectional cohort and it is important in the future to demonstrate that these findings can be replicated in a longitudinal study. The cohort in the study also has a wide range of age and athletic ability; although this was deliberate to allow study of athletes with a range of aerobic fitness. We have only studied the contribution of cardiac performance, and not ventilatory or neuromuscular function, to measures of aerobic fitness. All of the cardiac changes reported are during rest. Many of the strain parameters measured may not be independent and analysis of their individual interactions with aerobic fitness requires more complex modelling. We have not performed this in our study as very few strain parameters were associated with aerobic fitness.

\section{Conclusions}

This cohort of endurance athletes had lower LV circumferential strain, LV torsion and biventricular diastolic strain rates than controls. Increased LT, which is a major determinant of performance in endurance athletes, was associated with decreased LV torsion. Further work is needed to understand the mechanisms by which this occurs.

\section{Abbreviations}

CMR, cardiovascular magnetic resonance; CPX, cardiopulmonary exercise testing; EDV, end diastolic volume; EDVI, end diastolic volume indexed to body surface area; ESV, end systolic volume; LT, lactate threshold; LV, left ventricle; LVM, left ventricle mass; LVMI, left ventricle mass indexed to body surface area; $\mathrm{RV}$, right ventricle; SPAMM, spatial modulation of magnetization; SSFP, steady state free procession; $\dot{V} O 2$ max, maximal oxygen uptake $(\mathrm{ml} / \mathrm{min} / \mathrm{kg})$

\section{Acknowledgements}

Not applicable.

\section{Funding}

PS (FS/12/88/29901) \& SP (FS/1062/28409) are funded by British Heart Foundation fellowships. This study was supported by the National Institute for Health Research Leeds Clinical Research Facility. The views expressed are those of the author(s) and not necessarily those of the NHS, NIHR or the Department of Health.

\section{Availability of supporting data \\ Not applicable.}

\section{Authors' contributions}

PPS, Conception and design, analysis, interpretation of data, drafting of manuscript. BE, Analysis, critical and intellectual revision of manuscript. AKM, Analysis, critical and intellectual revision of manuscript. REL, Analysis, critical and intellectual revision of manuscript. GKL, Analysis, critical and intellectual revision of manuscript. LED, Critical and intellectual revision of manuscript. DPR, Critical and intellectual revision of manuscript. TAM, Critical and intellectual revision of manuscript. PG, Critical and intellectual revision of manuscript. CF, Interpretation of data, critical and intellectual revision of manuscript. JPG, Interpretation of data, critical and intellectual revision of manuscript. SP, Conception and design, interpretation of data, drafting of manuscript.

All authors read and approved the manuscript.

\section{Competing interests}

The authors declare that they have no competing interests.

\section{Consent for publication}

Not applicable.

\section{Ethics approval and consent to participate}

Ethical approval was given by the National Research Ethics Service Committee Yorkshire \& the Humber (14/YH/0126). All participants gave informed written consent.

\section{Author details}

${ }^{1}$ Multidisciplinary Cardiovascular Research Centre (MCRC) and Leeds Institute of Cardiovascular and Metabolic Medicine, University of Leeds, Clarendon 
Way, Leeds LS2 9JT, UK. ${ }^{2}$ Multidisciplinary Cardiovascular Research Centre (MCRC) and School of Biomedical Sciences, University of Leeds, Clarendon Way, Leeds LS2 9JT, UK.

Received: 28 April 2016 Accepted: 8 July 2016

Published online: 17 August 2016

\section{References}

1. Maron BJ, Pelliccia A. The heart of trained athletes: cardiac remodeling and the risks of sports, including sudden death. Circulation. 2006;114:1633-44.

2. Arbab-Zadeh A, Perhonen M, Howden E, et al. Cardiac remodeling in response to 1 year of intensive endurance training. Circulation. 2014;130:2152-61.

3. Utomi V, Oxborough D, Whyte GP, et al. Systematic review and metaanalysis of training mode, imaging modality and body size influences on the morphology and function of the male athlete's heart. Heart. 2013;99:1727-33.

4. Caselli S, Montesanti D, Autore C, et al. Patterns of left ventricular longitudinal strain and strain rate in Olympic athletes. J Am Soc Echocardiogr. 2015;28:245-53.

5. Nottin S, Doucende G, Schuster-Beck I, et al. Alteration in left ventricular normal and shear strains evaluated by 2D-strain echocardiography in the athlete's heart. J Physiol. 2008:586:4721-33.

6. Teske AJ, Prakken NH, De Boeck BW, et al. Echocardiographic tissue deformation imaging of right ventricular systolic function in endurance athletes. Eur Heart J. 2009;30:969-77.

7. Burns AT, McDonald IG, Thomas JD, et al. Doin' the twist: new tools for an old concept of myocardial function. Heart. 2008;94:978-83.

8. el Ibrahim SH. Myocardial tagging by cardiovascular magnetic resonance: evolution of techniques-pulse sequences, analysis algorithms, and applications. J Cardiovasc Magn Reson. 2011;13:36.

9. Shehata ML, Cheng S, Osman NF, et al. Myocardial tissue tagging with cardiovascular magnetic resonance. J Cardiovasc Magn Reson. 2009;11:55.

10. Young AA, Cowan BR. Evaluation of left ventricular torsion by cardiovascular magnetic resonance. J Cardiovasc Magn Reson. 2012;14:49.

11. Moody WE, Taylor RJ, Edwards NC, et al. Comparison of magnetic resonance feature tracking for systolic and diastolic strain and strain rate calculation with spatial modulation of magnetization imaging analysis. J Magn Reson Imaging. 2015;41:1000-12.

12. Balady GJ, Arena R, Sietsema K, et al. Clinician's Guide to cardiopulmonary exercise testing in adults: a scientific statement from the American Heart Association. Circulation. 2010;122:191-225.

13. Bentley DJ, Newell J, Bishop D. Incremental exercise test design and analysis: implications for performance diagnostics in endurance athletes. Sports Med. 2007;37:575-86.

14. Milliken MC, Stray-Gundersen J, Peshock RM, et al. Left ventricular mass as determined by magnetic resonance imaging in male endurance athletes. Am J Cardiol. 1988;62:301-5.

15. Scharhag J, Schneider G, Urhausen A, et al. Athlete's heart: right and left ventricular mass and function in male endurance athletes and untrained individuals determined by magnetic resonance imaging. J Am Coll Cardiol. 2002;40:1856-63.

16. Steding K, Engblom H, Buhre T, et al. Relation between cardiac dimensions and peak oxygen uptake. J Cardiovasc Magn Reson. 2010;12:8.

17. Messroghli DR, Bainbridge GJ, Alfakih $K$, et al. Assessment of regional left ventricular function: accuracy and reproducibility of positioning standard short-axis sections in cardiac MR imaging. Radiology. 2005;235:229-36.

18. Swoboda PP, Larghat A, Zaman A, et al. Reproducibility of myocardial strain and left ventricular twist measured using complementary spatial modulation of magnetization. J Magn Reson Imaging. 2013:39(4):887-94.

19. Rossiter $\mathrm{HB}$, Kowalchuk JM, Whipp BJ. A test to establish maximum $\mathrm{O} 2$ uptake despite no plateau in the $\mathrm{O} 2$ uptake response to ramp incremental exercise. J Appl Physiol (1985). 2006;100:764-70.

20. Hansen JE, Sue DY, Wasserman K. Predicted values for clinical exercise testing. Am Rev Respir Dis. 1984;129:549-55.

21. Osborne G, Wolfe LA, Burggraf GW, et al. Relationships between cardiac dimensions, anthropometric characteristics and maximal aerobic power (VO2max) in young men. Int J Sports Med. 1992;13:219-24.

22. Bassett Jr DR, Howley ET. Limiting factors for maximum oxygen uptake and determinants of endurance performance. Med Sci Sports Exerc. 2000;32:70-84.

23. Scott JM, Esch BT, Haykowsky MJ, et al. Effects of high intensity exercise on biventricular function assessed by cardiac magnetic resonance imaging in endurance trained and normally active individuals. Am 」 Cardiol. 2010;106:278-83.

24. Esch BT, Scott JM, Haykowsky MJ, et al. Changes in ventricular twist and untwisting with orthostatic stress: endurance athletes versus normally active individuals. J Appl Physiol (1985). 2010;108:1259-66.

25. Stohr EJ, McDonnell B, Thompson J, et al. Left ventricular mechanics in humans with high aerobic fitness: adaptation independent of structural remodelling, arterial haemodynamics and heart rate. J Physiol. 2012:590:2107-19

26. Stewart $\mathrm{GM}$, Yamada $\mathrm{A}$, Haseler $\mathrm{L}$, et al. Altered ventricular mechanics after $60 \mathrm{~min}$ of high-intensity endurance exercise: insights from exercise speckletracking echocardiography. Am J Physiol Heart Circ Physiol. 2015;308:H875-83.

27. Weiner RB, Hutter Jr AM, Wang F, et al. The impact of endurance exercise training on left ventricular torsion. JACC Cardiovasc Imaging. 2010;3:1001-9.

28. van Dalen BM, Kauer F, Vletter WB, et al. Influence of cardiac shape on left ventricular twist. J Appl Physiol (1985). 2010;108:146-51.

29. Kenney WL, Hodgson JL. Variables predictive of performance in elite middle-distance runners. Br J Sports Med. 1985;19:207-9.

30. Pereira F, de Moraes $R$, Tibirica E, et al. Interval and continuous exercise training produce similar increases in skeletal muscle and left ventricle microvascular density in rats. Biomed Res Int. 2013;2013:752817.

31. Hafstad AD, Boardman NT, Lund J, et al. High intensity interval training alters substrate utilization and reduces oxygen consumption in the heart. J Appl Physiol (1985). 2011;111:1235-41.

32. Stuber M, Scheidegger MB, Fischer SE, et al. Alterations in the local myocardial motion pattern in patients suffering from pressure overload due to aortic stenosis. Circulation. 1999;100:361-8.

33. Chung J, Abraszewski P, Yu X, et al. Paradoxical increase in ventricular torsion and systolic torsion rate in type I diabetic patients under tight glycemic control. J Am Coll Cardiol. 2006;47:384-90.

34. Baggish AL, Wang F, Weiner RB, et al. Training-specific changes in cardiac structure and function: a prospective and longitudinal assessment of competitive athletes. J Appl Physiol (1985). 2008;104:1121-8.

35. Pluim BM, Zwinderman AH, van der Laarse A, et al. The athlete's heart. A metaanalysis of cardiac structure and function. Circulation. 2000;101:336-44.

\section{Submit your next manuscript to BioMed Central and we will help you at every step:}

- We accept pre-submission inquiries

- Our selector tool helps you to find the most relevant journal

- We provide round the clock customer support

- Convenient online submission

- Thorough peer review

- Inclusion in PubMed and all major indexing services

- Maximum visibility for your research

Submit your manuscript at www.biomedcentral.com/submit
) Biomed Central 